\title{
Publisher Correction: Similarity-driven multi-view embeddings from high-dimensional biomedical data
}

\author{
Brian B. Avants (D), Nicholas J. Tustison (D) and James R. Stone
}

Correction to: Nature Computational Science https://doi.org/10.1038/s43588-021-00029-8, published online 22 February 2021.

In the version of this Article originally published, the PTBP database containing brain age data, and the code capsule used to generate the simulation data and the visualized plots in the main manuscript, were mistakenly omitted from the reference list. References for these have now been added to the Article and are cited in the 'Data availability' and 'Code availability' sections.

80. Avants, B. B., Tustison, N. J. \& Stone, J. R. SiMLR in ANTsR: interpretable, similarity-driven multi-view embeddings from high-dimensional biomedical data. Code Ocean https://doi.org/10.24433/CO.3087836.v2 (2021).

81. Avants, B. B., Tustison, N. J. \& Wang, D. J. J. The pediatric template of brain perfusion (PTBP). figshare https://doi.org/10.6084/ m9.figshare.923555.v20 (2013).

In addition, in the Acknowledgements the text 'This work is supported by the Office of Naval Research (N00014-18-1-2440) and National Institutes of Health (K01-ES025432-01)' was incorrect; it should have read 'This work is supported by a combined grant from Cohen Veterans Bioscience (CVB-461) and the Office of Naval Research (N00014-18-1-2440) as well as the National Institutes of Health (K01-ES025432-01)'. This has now been corrected.

Published online: 4 March 2021

https://doi.org/10.1038/s43588-021-00049-4

(c) The Author(s), under exclusive licence to Springer Nature America, Inc. 2021 